\title{
THE
}

\section{Universal target-enrichment baits for anthozoan (Cnidaria) phylogenomics: New approaches to long-standing problems}

Andrea M. Quattrini

Brant C. Faircloth

Luisa F. Dueñas

Tom C.L. Bridge

Mercer R. Brugler

See next page for additional authors

Follow this and additional works at: https://digitalcommons.uri.edu/bio_facpubs

The University of Rhode Island Faculty have made this article openly available. Please let us know how Open Access to this research benefits you.

This is a pre-publication author manuscript of the final, published article.

Terms of Use

This article is made available under the terms and conditions applicable towards Open Access Policy Articles, as set forth in our Terms of Use.

\section{Citation/Publisher Attribution}

Quattrini, AM, Faircloth, BC, Dueñas, LF, et al. Universal target-enrichment baits for anthozoan (Cnidaria) phylogenomics: New approaches to long-standing problems. Mol Ecol Resour. 2018; 18: 281- 295. https://doi.org/10.1111/1755-0998.12736

Available at: https://doi.org/10.1111/1755-0998.12736 


\section{Authors}

Andrea M. Quattrini, Brant C. Faircloth, Luisa F. Dueñas, Tom C.L. Bridge, Mercer R. Brugler, Iván F. CaliztoBotía, Danielle M. DeLeo, Sylvain Forêt, Santiago Herrera, Simon M.Y. Lee, David J. Miller, Carlos Prada, Gandhi Rádis-Baptista, Catalina Ramirez-Portilla, Juan A. Sánchez, Estefanía Rodriguez, and Catherine S. McFadden 
2 DR. ANDREA M. QUATTRINI (Orcid ID : 0000-0002-4247-3055)

3 DR. BRANT FAIRCLOTH (Orcid ID : 0000-0002-1943-0217)

4 MR. SANTIAGO HERRERA (Orcid ID : 0000-0001-7204-9434)

5 DR. DAVID MILLER (Orcid ID : 0000-0003-0291-9531)

8 Article type : Resource Article

11 Universal target-enrichment baits for anthozoan (Cnidaria) phylogenomics: New approaches to 12 long-standing problems

A.M. Quattrini ${ }^{1 *}$, B.C. Faircloth ${ }^{2}$, L.F. Dueñas ${ }^{3}$, T.C.L. Bridge ${ }^{4,5}$, M.R. Brugler ${ }^{6,7}$, I.F. Calixto16 Botía $^{3,8}$, D.M. DeLeo ${ }^{9,10}$, S. Forêt ${ }^{11}$, S. Herrera ${ }^{12}$, S. Lee ${ }^{13}$, D.J. Miller ${ }^{5}$, C. Prada ${ }^{14}$, G. Rádis-

17 Baptista $^{15}$, C. Ramírez-Portilla ${ }^{3,8}$, J.A. Sánchez ${ }^{3}$, E. Rodríguez ${ }^{6}$, and C.S. McFadden ${ }^{1}$

$19{ }^{1}$ Department of Biology, Harvey Mudd College, 1250 N Dartmouth Ave, Claremont, CA 91711, 20 USA

$21{ }^{2}$ Department of Biological Sciences and Museum of Natural Science, Louisiana State University, 22 Baton Rouge, LA 70803, USA

$23{ }^{3}$ Departamento de Ciencias Biológicas-Facultad de Ciencias, Laboratorio de Biología Molecular 24 Marina (BIOMMAR), Universidad de los Andes, Carrera 1 No. 18A - 12, (J 212, J308 Lab), 25 Bogotá, Colombia

$26{ }^{4}$ Queensland Museum Network, 70-102 Flinders St, Townsville, Queensland 4810, Australia

$27{ }^{5}$ Australian Research Council Centre of Excellence for Coral Reef Studies, James Cook

28 University, Townsville, Queensland 4811, Australia

This is the author manuscript accepted for publication and has undergone full peer review but has not been through the copyediting, typesetting, pagination and proofreading process, which may lead to differences between this version and the Version of Record. Please cite this article as doi: $\underline{10.1111 / 1755-0998.12736}$

This article is protected by copyright. All rights reserved 
$29{ }^{6}$ Division of Invertebrate Zoology, American Museum of Natural History, Central Park West at 30 79th Street, New York NY 10024, USA

$31{ }^{7}$ Biological Sciences Department, NYC College of Technology, City University of New York,

32 Brooklyn NY 11201, USA

$33{ }^{8}$ Department of Animal Ecology and Systematics, Justus Liebig Universität, Heinrich-Buff-Ring

34 26-32 IFZ D-35392, Giessen, Germany

$35{ }^{9}$ Department of Biological Sciences, Florida International University, 3000 NE 151 Street, North

36 Miami, FL 33181, USA

$37{ }^{10}$ Biology Department, Temple University, 1900 N $12^{\text {th }}$ St., Philadelphia PA, 19122 USA

$38{ }^{11}$ Research School of Biology, Australian National University, Canberra, ACT0200, Australia

$39{ }^{12}$ Department of Biological Sciences, Lehigh University, Bethlehem, PA 18015 USA

$40 \quad{ }^{13}$ State Key Laboratory of Quality Research in Chinese Medicine and Institute of Chinese

41 Medical Sciences, University of Macau, Macao China

$42{ }^{14}$ Department of Biological Sciences, University of Rhode Island, Kingston, RI 02881

$43{ }^{15}$ Institute for Marine Sciences, Federal University of Ceara, Av da Abolição 3207, Fortaleza/CE,

$44 \quad 60165081$, Brazil

45

46

47 *Corresponding author: aquattrini@g.hmc.edu

50 Keywords: coral, ultraconserved elements, UCE, target-capture, exon, phylogeny

51 Running Title: Target-enrichment for anthozoan phylogenomics

Abstract

Anthozoans (e.g., corals, anemones) are an ecologically important and diverse group of

57 marine metazoans that occur from shallow to deep waters worldwide. However, our

58 understanding of the evolutionary relationships among the $\sim 7500$ species within this class is

59 hindered by the lack of phylogenetically informative markers that can be reliably sequenced 
across a diversity of taxa. We designed and tested 16,306 RNA baits to capture 720

61 Ultraconserved Element loci and 1,071 exon loci. Library preparation and target enrichment was

62 performed on 33 taxa from all orders within the class Anthozoa. Following Illumina sequencing

63 and Trinity assembly, we recovered 1,774 of 1,791 targeted loci. The mean number of loci

64 recovered from each species was $638 \pm 222$, with more loci recovered from octocorals $(783 \pm$

65138 loci) than hexacorals ( $475 \pm 187$ loci). Parsimony informative sites ranged from $26-49 \%$ for

66 alignments at differing hierarchical taxonomic levels (e.g., Anthozoa, Octocorallia,

67 Hexacorallia). The percent of variable sites within each of three genera (Acropora, Alcyonium,

68 and Sinularia) for which multiple species were sequenced ranged from 4.7-30\%. Maximum

69 likelihood analyses recovered highly resolved trees with topologies matching those supported by

70 other studies, including the monophyly of the order Scleractinia. Our results demonstrate the

71 utility of this target-enrichment approach to resolve phylogenetic relationships from relatively

72 old to recent divergences. Re-designing the baits with improved affinities to capture loci within

73 each sub-class will provide a valuable toolset to address systematic questions, further our

74 understanding of the timing of diversifications, and help resolve long-standing controversial

75 relationships in the class Anthozoa.

76

77

Introduction

79

80

Anthozoan cnidarians play critical roles in many marine ecosystems. The class contains $\sim 7,500$ extant species (i.e., soft corals, sea fans, stony corals, black corals, and anemones) that

82 live worldwide in a variety of marine habitats-from tropical shallow waters to the cold, deep

83 sea (Daly et al. 2007). Classification of Anthozoa has traditionally been based on morphological

84 characters such as skeletal morphology, colony organization and soft-tissue anatomy of the

85 polyps (Daly et al. 2007), including the arrangement of internal mesenteries (Fautin and Mariscal

86 1991). Long-standing views have recognized the anthozoan sub-classes Octocorallia and

87 Hexacorallia as reciprocally monophyletic (Daly et al. 2007), a view also supported by recent

88 phylogenomic analyses of 10s to 100s of genes (Zapata et al. 2015; Pratlong et al. 2017). Within

89 each sub-class, however, molecular phylogenetic studies have revealed widespread homoplasy in

90 morphological characters and widespread polyphyly at the ordinal, sub-ordinal, family, and

This article is protected by copyright. All rights reserved 
91 genus levels (e.g., Fukami et al. 2008; McFadden et al. 2010; Rodríguez et al. 2014; Daly et al.

92 2017). Consequently, deep flaws exist in our understanding of the phylogenetic relationships

93 among and within anthozoan orders. Attempts to resolve the deep phylogenetic relationships

94 among anthozoans using molecular data have largely been unsuccessful due to relatively slow

95 evolutionary rates of mitochondrial genomes (Shearer et al. 2002; Hellberg 2006; Huang et al.

96 2008; Forsman et al. 2009), lack of signal in rDNA (Berntson et al. 2001; Daly et al. 2003) and

97 difficulty in identifying and developing PCR primers for single-copy nuclear genes that can be

98 amplified across the entire class (McFadden et al. 2011).

99 Within most anthozoan orders, there is also a lack of phylogenetic resolution at the

100 species level. This may be due to incomplete lineage sorting in gene trees, insufficient data due

101 to the small number of currently available markers, hybridization, and/or lack of morphological

102 synapomorphies in taxonomy (McFadden et al. 2010, 2011, 2017; Prada et al. 2014; Rodríguez

103 et al. 2014; Grajales and Rodríguez 2016; Daly et al. 2017). Currently available markers are

104 insufficient at resolving species boundaries for the majority of anthozoans. For octocorals, an

105 extended mitochondrial barcode $(C O I+i g r l+m t M u t S)$ has proven useful for revealing cryptic

106 species and delimiting species boundaries within some clades; however, the divergence criterion

107 proposed (McFadden et al. 2011) to elucidate these boundaries is low (>0.5\% p-distance) and

108 often no genetic divergence is observed among congeneric species (McFadden et al. 2011,

109 Dueñas et al. 2014, Pante et al. 2015). The low genetic variability in the mitochondrial genome

110 has been attributed to a unique mis-match repair enzyme (mtMutS) that potentially repairs

111 mutations (Bilewitch and Degnan 2011) thereby causing reduced mitochondrial sequence

112 variation in octocorals when compared to other metazoans (Shearer et al. 2002). Mitochondrial

113 sequence variation is also low in the hexacorals (Hellberg et al. 2006; Daly et al. 2010), creating

114 difficulties in resolving species boundaries using traditional mitochondrial barcodes (i.e., COI,

115 Hebert et al. 2003; Shearer and Coffroth 2008). Although several studies have resolved species

116 boundaries using a nuclear ITS marker (e.g., Medina et al. 1999; Pinzon and LaJeunesse 2011),

117 using ITS poses problems as it is not a single-locus marker (Vollmer and Palumbi 2004) and

118 there are often high levels of intra-specific variation (Van Oppen et al. 2000). Methods that

119 allow for collecting and analyzing numerous loci across shallow and deep levels of divergence

120 are sorely needed.

121 NGS-based methods that have been developed to enable the capture of large numbers of 
122 homologous loci in large-scale phylogenetic studies include amplicon sequencing, restriction 123 site-associated DNA (RADseq) methods, transcriptome sequencing and target enrichment of 124 genomic DNA (see McCormack et al. 2013a). Although RADSeq is an effective approach for 125 species-level phylogenetics and species delimitation within anthozoan genera (e.g., Combosch 126 and Vollmer 2015; Pante et al. 2015; Herrera and Shank 2016; McFadden et al. 2017; Johnston 127 et al. 2017), using RADseq to address deeper-level relationships is not feasible due to locus drop 128 out (Althoff et al. 2007; McCormack et al. 2013a). Transcriptomic data have been used to 129 reconstruct deep relationships within Cnidaria (Zapata et al. 2015; Pratlong et al. 2017), but the 130 need for RNA limits the use of this method to taxa for which fresh material can be collected and 131 preserved appropriately. Alternatively, target enrichment of ultraconserved elements (UCEs) 132 (Faircloth et al. 2012) has proven robust in inferring species histories of both vertebrates [e.g., 133 fishes (Faircloth et al. 2013), birds (McCormack et al. 2013b), reptiles (Crawford et al. 2012), 134 and mammals (McCormack et al. 2012)] and invertebrates [e.g., arachnids (Starrett et al. 2016), 135 hymenopterans (Branstetter et al. 2017), and coleopterans (Baca et al. 2017)] across shallow to 136 deep timescales. UCEs occur in high numbers throughout genomes across the tree of life, 137 including Cnidaria (Ryu et al. 2012), making them easy to identify and align among divergent 138 species (Faircloth et al. 2012). As the name implies, UCEs are highly conserved regions of the 139 genome, but the flanking regions surrounding UCEs are more variable and phylogenetically 140 informative (Faircloth et al. 2012). Some advantages of using target enrichment of UCEs include 141 that 100 s to 1000 s of loci can be sequenced at a relatively low cost from a wide range of taxa 142 (Faircloth et al. 2012); they can be generated from 100 year old, formalin-preserved museum 143 specimens and specimens with degraded DNA (McCormack et al. 2016; Ruane and Austin 144 2017); and they have proven useful at resolving evolutionary questions across both shallow and 145 deep time scales (Smith et al. 2013; McCormack et al. 2013b; Manthey et al. 2016). Similar 146 approaches using target-enrichment of coding regions, or exon capturing (Bi et al. 2012; Ilves 147 and López-Fernández 2014; Hugall et al. 2016), have also proven valuable in phylogenomics. 148 We used all available genomes and transcriptomes to design a set of target-capture baits 149 for enriching both UCEs and exons for use in anthozoan phylogenetics. Herein, we discuss how 150 loci were targeted and baits were designed. Using an in silico analysis, we demonstrate that these 151 loci recover the established sub-class and ordinal relationships among anthozoans. Finally, we 152 test the utility of these baits in vitro using 33 species from across both sub-classes of Anthozoa. 


\section{Materials and Methods}

155 Preparation of Genomes and Transcriptomes

156 Genomic and transcriptomic data were gathered from various sources for use in bait

157 design and in silico testing (Table S1). All data were masked for repetitive regions,

158 retroelements, small RNAs, and transposons using Repeat Masker open-4.0 (Smit et al. 2015).

159 The N50 was calculated for each genome using stats.sh in the BBtools package (Bushnell 2015).

160 We then constructed 2bit files for all genomes and transcriptomes (faToTwoBit, BLAT Suite,

161 Kent 2002) and simulated 100 bp paired reads from each genome and transcriptome using the

162 program art_illumina (Huang et al. 2012) in order to map simulated reads back to the genomes.

163 All programs and parameters used for the entire workflow can be found in Supplemental File 1.

Identification of UCE Loci and Bait Design

166 We used the open-source program PHYLUCE (Faircloth 2016) and followed the

167 workflow in the online tutorial (http://phyluce.readthedocs.io/en/latest/tutorial-four.html), with a

168 few modifications to identify conserved regions and design baits to target these regions for

169 downstream next-generation sequencing (Faircloth 2017). We first aligned an average of 34

170 million, 100 bp simulated-reads from each of the four exemplar taxa, Acropora digitifera,

171 Exaiptasia pallida, Renilla muelleri, and Pacifigorgia irene, to a base genome, Nematostella

172 vectensis. Nematostella vectensis ('nemve') was chosen as the base genome for the primary bait

173 design because it is one of the most well assembled and annotated anthozoan genomes. We used

174 stampy v. 1(Lunter and Goodson 2011), with a substitution rate set at 0.05, to map conserved

175 regions of each read-simulated genome to the base genome. Across all taxa, 0.6 to $1.8 \%$ of the

176 reads mapped to the nemve genome. The resulting alignment file was transformed from SAM

177 format into BAM format (samtools, Li et al. 2009) and then transformed into a BED formatted

178 file (BEDtools, Quinlan and Hall 2010). These BED files were sorted by scaffold/contig and then

179 by position along that scaffold/contig. We then merged together the alignment positions in each

180 file that were close ( $<100 \mathrm{bp}$ ) to one another using bedtools. In addition, sequences that included

181 masked regions $(>25 \%)$ or ambiguous ( $\mathrm{N}$ or X) bases or were too short (<80 bp) were removed

182 using phyluce_probe_strip_masked_loci_from_set. These steps resulted in BED files containing

183 regions of conserved sequences shared between nemve and each of the exemplar taxa for further 
184 analysis. An SQLite table was created using phyluce_probe_get_multi_merge_table, and 185 included 70,312 loci that were shared between pairs of taxa.

186 We queried the SQLite table and output a list of 1,794 conserved regions found in nemve 187 and the other four exemplar taxa using phyluce_probe_query_multi_merge_table. This list plus 188 phyluce_probe_get_genome_sequences_from_bed was used to extract the conserved regions 189 from the nemve genome. These regions were buffered to $160 \mathrm{bp}$ by including an equal amount of $190 \quad 5^{\prime}$ and $3^{\prime}$ flanking sequence from the nemve genome. Another filter was performed at this stage to 191 remove sequences < $160 \mathrm{bp}$, sequences with $>25 \%$ masked bases, or sequences with ambiguous 192 bases. A temporary set of sequence capture baits was designed from the loci found in this final 193 FASTA file. Using phyluce_probe_get_tiled_probes, we designed the bait set by tiling two 120 194 bp baits over each locus that overlapped in the middle by $40 \mathrm{bp}$ (3X density). This temporary set 195 of baits was screened to remove baits with $>25 \%$ masked bases or high $(>70 \%)$ or low $(<30 \%)$ 196 GC content. Any potential duplicates were also removed using phyluce_probe_easy_lastz and 197 phyluce_probe_remove_duplicate_hits_from_probes_using_lastz. Bait sequences were considered duplicates if they were $\geq 50 \%$ identical over $\geq 50 \%$ of their length.

199 The temporary bait set (2,131 baits, targeting 1,787 loci) was aligned back to nemve and 200 the four exemplar taxa using phyluce_probe_run_multiple_lastzs_sqlite, with an identity value of $20170 \%$ (the minimum sequence identity for which a bait could be an accepted match to the 202 genome) and a minimum coverage of $83 \%$ (default value). From these alignments, baits that 203 matched multiple loci were removed. We then extracted $180 \mathrm{bp}$ of the sequences from the 204 alignment files and input the data into FASTA files using

205 phyluce_probe_slice_sequence_from_genomes. A list containing 710 loci found in at least three 206 of the taxa was created. Based on this list of 710 loci, the anthozoan UCE bait set was re207 designed to target these 710 loci using phyluce_probe_get_tiled_probe_from_multiple_inputs, 208 nemve, and the four exemplar genomes. Using this script, 120-bp baits were tiled (3X density, 209 middle overlap) and screened for high $(>70 \%)$ or low $(<30 \%)$ GC content, masked bases

$210(>25 \%)$, and duplicates. This bait set included a total of 5,459 non-duplicated baits targeting 710 211 anthozoan loci. All above methods were repeated to produce additional octocoral-specific baits 212 and capture octocoral-specific loci. We repeated the above analyses using $R$. muelleri as the base 213 genome and P. irene, Paragorgia stephencairnsi, and Antillogorgia bipinnata as the exemplar 214 taxa to add 1,317 baits targeting an additional 168 UCE loci to the dataset. 
Identification of Exon Loci and Bait Design

217 To design baits to target exon regions, the above methods were repeated using available

218 transcriptome data. An average of 7 million reads from five exemplar transcriptome-enabled taxa

219 (A. digitifera, Cerianthidae, Edwardsiella lineata, Gorgonia ventalina, and Paramuricea sp.)

220 were simulated and 1.1 to $15.3 \%$ of these reads per species were aligned to the nemve

221 transcriptome. After we converted the alignments to BED files, merged overlapping reads, and

222 filtered data for short loci and repetitive regions, 44,215 conserved sequences were added to an

223 SQLite database. We queried this database and selected 3,700 loci that were found in nemve and

224 the additional five exemplar taxa. Following a second screening for masked regions, high/low

225 GC content, and duplicates, a temporary exon bait set (5,661 baits) targeting 3,633 exon loci was

226 designed. The temporary baits were re-aligned to the transcriptomes of nemve and the additional

227 five exemplar anthozoans to ensure we could locate the loci. A set of 906 loci that were found in

228 nemve and the additional five exemplar anthozoans were added to an SQLite database. We re-

229 designed the exon bait set to target these 906 exon loci using

230 phyluce_probe_get_tiled_probe_from_multiple_inputs, nemve, and the five exemplar

231 transcriptomes. This bait set included a total of 8,080 non-duplicated baits targeting 906 loci

232 across all anthozoans. To add more octocoral-specific baits and loci, we then repeated the above

233 analyses with Paramuricea sp. as the base transcriptome and Anthomastus sp., Corallium

234 rubrum, Eunicea flexuosa, G. ventalina, Keratoisidinae sp., and Nepthyigorgia sp. as the

235 exemplar taxa to add 4,914 baits targeting an additional 407 loci to the dataset.

Final Bait Screening

All of the bait sets designed with various sets of data as described above (see Table S1) were screened against one another to remove redundant baits ( $\geq 50 \%$ identical over $>50 \%$ of their

240 length), allowing us to create a final non-duplicated Anthozoa bait set. We also screened these

241 baits (70\% identity, 70\% coverage) against the Symbiodinium minutum genome by using

242 phyluce_probe_run_multiple_lastzs_sqlite and phyluce_probe_slice_sequence_from_genomes

243 and removed loci that matched the symbiont. Bait names in the final bait FASTA file begin with

244 'uce-' if designed using genomes to target UCEs and 'trans-' if designed using transcriptomes to 245 target exons.

This article is protected by copyright. All rights reserved 
248 In silico tests were performed to check how well the designed baits aligned to existing 249 genomes and transcriptomes. First, phyluce_probe_run_multiple_lastzs_sqlite was used to align

250 the UCE baits to the nine 2-bit formatted genomes and an outgroup genome (Hydra

251 magnipapillata) and the exon baits to the 24 2-bit formatted transcriptomes (Table S1). An

252 identity value of 50\% was chosen for alignments (following the PHYLUCE tutorial). For each

253 bait test, the matching FASTA data were sliced out of each genome or transcriptome, plus 200

254 bp of 5' and 3' flanking regions, using phyluce_probe_slice_sequence_from_genomes. This

255 resulted in an average of $429 \pm 178 \mathrm{SD}$ (44 to 599 per species) UCE loci and $497 \pm 230$ SD (206

256 to 857) exon loci per anthozoan species (Table 1). To do a final screen for duplicates, loci were

257 matched back to the baits using phyluce_assembly_match_contigs_to_probes, with a minimum

258 coverage of $67 \%$ and minimum identity of $80 \%$ (default values following the PHYLUCE

259 tutorial). Here, an average of $355 \pm 166$ SD (25 to 529 per species) non-duplicate UCE loci and

$260354 \pm 210$ SD (106 to 670) non-duplicate exon loci were recovered per anthozoan species (Table

261 1). Each locus was exported into a FASTA file and aligned with MAFFT (Katoh et al. 2002)

262 using phyluce_align_seqcap_align with default parameters.

263 The resulting alignments were trimmed internally using GBlocks (Castresana 2000,

264 Talavera and Castresana 2007)

265 using phyluce_align_get_gblocks_trimmed_alignments_from_untrimmed with default

266 parameters. Two final datasets were then created using

267 phyluce_align_get_only_loci_with_min_taxa, in which all locus alignments contained at least 4

268 of the 10 taxa for the genome data and 9 of the 24 taxa for the transcriptome data. We then

269 concatenated the resulting alignments into separate supermatrices; one containing UCE loci from

27010 genome-enabled taxa and the other containing exon loci from the 24 transcriptome-enabled

271 taxa. Maximum likelihood (ML) inference was conducted on each supermatrix using RAxML v8

272 (Stamatakis 2014). This analysis was carried out using rapid bootstrapping, which allows for a

273 complete analysis (20 ML searches and 200 bootstrap replicates) in one step. We also conducted

274 a Bayesian inference (10 million generations, 35\% burnin) using ExaBayes (Aberer et al. 2014).

275 An extended majority rule consensus tree was produced. A General-Time Reversible model of 
nucleotide substitution with a gamma distributed rate variation (GTRGAMMA) was used in both

277 ML and Bayesian analyses.

Following the in silico test, the list of designed baits was sent to MYcroarray for synthesis. MYcroarray further screened and removed baits that either had repetitive elements or the potential to cross-hybridize ( $0.007 \%$ total baits removed). We then tested the bait set on 33 anthozoan specimens (Table 2), with both sub-classes and all major orders and sub-orders (for Octocorallia) represented. DNA from these specimens included recent extractions from tissue that had been stored frozen (in liquid nitrogen) for $25 \mathrm{yrs}$ or in $95 \% \mathrm{EtOH}$ for up to $10 \mathrm{yrs}$, as well as extractions that had been stored frozen $\left(-20^{\circ} \mathrm{C}\right)$ for $10 \mathrm{yrs}$ (see Table S2).

DNA was extracted using a Qiagen DNeasy Blood \& Tissue kit, Qiagen Gentra Kit, or a CTAB extraction protocol (McFadden et al. 2006). DNA quality was assessed using a Nanodrop spectrophotometer, with 260/280 ratios ranging from 1.8-2.1 and 260/230 ratios ranging from

291 the majority of samples, we then sheared approximately $600 \mathrm{ng}$ DNA (10 ng per $\mu \mathrm{L})$ to a target 292 size range of 400-800 bp using sonication (Q800R QSonica Inc. Sonicator). For eight samples 293 (Table 2), we sheared $35 \mu \mathrm{L}$ (115-372 ng, average $217 \mathrm{ng}$ ) of EDTA-free DNA using enzymes 294 from the Kapa HyperPlus (Kapa Biosystems) library preparation kit. These samples were mixed 295 on ice with $5 \mu \mathrm{L}$ of Kapa Frag buffer and $10 \mu \mathrm{L}$ of the Kapa Frag enzyme and put on a pre296 cooled $\left(4^{\circ} \mathrm{C}\right)$ thermocycler prior to incubation for $10-15 \mathrm{~min}$ at $37^{\circ} \mathrm{C}$ to achieve a target size 297 range of 400-800 bp. After shearing, DNA was run out on a 1\% agarose gel (120V, $60 \mathrm{~min}$ ).

298 Small DNA fragments were removed from each sample (250 ng DNA) using a generic SPRI 299 substitute (Rohland and Reich 2012; Glenn et al. 2016) bead cleanup (3X). DNA was re-

300 suspended in $25 \mu \mathrm{L}$ double-distilled water ( $\mathrm{ddH} 20$ ).

301 Details of library preparation and target enrichment can be found in Supplemental File 2.

302 Briefly, library preparation (Kapa Biosystems) was carried out on the majority of DNA samples

303 (Table 2) using a Kapa Hyper Prep protocol. For the subset of the samples for which DNA was

304 sheared using enzymes (Table 2), we followed the protocol in the Kapa Hyper Plus enzyme-

305 shearing library preparation kit (Kapa Biosystems). Universal Y-yoke oligonucleotide adapters and custom iTru dual-indexed primers were used in library preparations (Glenn et al. 2016). For 
target enrichment, the MYcroarray MyBaits were diluted in 1/2 (250 ng) of the standard (500 ng)

308 MyBaits reaction, using $2.5 \mu \mathrm{L}$ of the baits and $2.5 \mu \mathrm{L}$ of ddH20 for all samples. Different bait

309 strengths were tested on a set of six samples (Table 2): full bait strength (500 ng), 1/2 bait

310 strength ( $250 \mathrm{ng}), 1 / 4$ bait strength (125 ng), and 1/8 strength (63 ng). One combined pool of all

311 enriched libraries was sent to Oklahoma Medical Research Facility for sequencing on 2/3 of a

312 lane of Illumina HiSeq 3000 (150bp PE reads).

313

314 Post-Sequencing Analyses

315 De-multiplexed Illumina reads were processed using PHYLUCE following the workflow

316 in the online tutorial (http://phyluce.readthedocs.io/en/latest/tutorial-one.html/), with a few

317 modifications (Suppl. File 1). The reads were first trimmed using the Illumiprocessor wrapper

318 program (Faircloth 2012) with default values and then assembled using Trinity v. 2.0 (Haas et al.

319 2013). We also assembled the data using Abyss 2.0 (Simpson et al. 2009) with a kmer value of

32031 . UCE and exon bait sequences were then separately matched to the assembled contigs (70\%

321 identity, 70\% coverage) using phyluce_assembly_match_contigs_to_probes to locate the loci.

322 Loci were then extracted using phyluce_assembly_get_match_counts and

323 phyluce_assembly_get_fastas_from_match_counts, exported into separate FASTA files and

324 aligned with default parameters using phyluce_align_seqcap_align, which uses MAFFT. Loci

325 were internally trimmed with GBlocks using

326 phyluce_align_get_gblocks_trimmed_alignments_from_untrimmed with default parameters.

327 Data matrices of locus alignments were created using

328 phyluce_align_get_only_loci_with_min_taxa, in which each locus had either 25\% or 50\%

329 species occupancy. Concatenated locus alignments consisted of exon loci only, UCE loci only,

330 and all loci. The number of parsimony informative sites was calculated for each alignment across

331 various taxonomic datasets. The script phyluce_align_get_informative_sites was used on the

332 following taxonomic datasets: Anthozoa+genome+outgroup (33 taxa used in in vitro test, plus

333 nine genome-enabled taxa and the outgroup H. magnipapillata), Anthozoa (33 taxa used in in

334 vitro test), Hexacorallia only (17 taxa used in in vitro test), and Octocorallia only (16 taxa used

335 in in vitro test). The total number of variable sites, total number of parsimony informative sites

336 and number of parsimony informative sites per locus were calculated. We also calculated the

337 total number of variable sites and the number of variable sites per locus for alignments 
338 containing species in each of three genera: Acropora (A. digitifera, A. millepora, A. muricata),

339 Alcyonium (A. acaule, A. digitatum, A. haddoni), and Sinularia (S. slieringsi, S. lochmodes, S.

340 maxima). For the three Acropora species, we used loci from one target-capture enrichment

341 sample and from the two Acropora genomes that were available.

342 ML inference was conducted on each alignment (exon loci only, UCE loci only, and all

343 loci) for the Anthozoa+genome+outgroup taxon set using RAxML v8. This analysis was carried

344 out using rapid bootstrapping, which allows for a complete analysis (20 ML searches and 200

345 bootstrap replicates) in one step. We also conducted a Bayesian analysis (10 million generations,

$34635 \%$ burnin) on the 25 and 50\% all-loci datasets using ExaBayes (Aberer et al. 2014). An

347 extended majority rule consensus tree was produced. A GTRGAMMA model was used in both

$348 \quad$ ML and Bayesian analyses.

349

350 Results

351 Identification of Loci and Bait Design

352 A total of 16,306 baits were designed to capture 1,791 anthozoan loci with 4 to 10 baits

353 targeting each locus. The principal UCE bait set included 5,513 baits designed to target 720 loci.

354 The principal exon bait set included 10,793 baits to target 1,071 loci. Four loci that matched

355 genomic regions in Symbiodinium minutum were removed from the dataset. These loci, however,

356 were also detected in azooxanthellate anthozoans, such as Chrysogorgia tricaulis.

In Silico Test

359 We generated two alignment matrices, one consisting of the exon loci taken from the

360 transcriptome-enabled taxa and the other one consisting of the UCE loci taken from the genome-

361 enabled taxa. The alignment matrix generated with the UCE loci, which included the $H$.

362 magnipapillata outgroup, had a total of 522 loci, with a trimmed mean locus length of $373 \mathrm{bp}$

363 (95\% CI: 8.4) and a total alignment length of 138,778 bp. The alignment matrix generated with

364 the exon loci included 407 loci, with a trimmed mean locus length of 462 bp (95\% CI: 5.8) and a

365 total length of 220,139 bp. The ML phylogenies generated from these alignments were well

366 supported and recovered monophyletic sub-classes and established ordinal relationships (Fig. 1).

367 The phylogeny generated with the UCE loci had 100\% support at all the nodes (Fig. 1a) whereas

368 the phylogeny generated with the exon loci had complete support at the majority (86\%) of the 
nodes (Fig. 1b). Trees produced using Bayesian inference were congruent with ML results.

372 The total number of reads obtained from Illumina sequencing ranged from 460,724 to

$37317,283,798$ reads per sample (mean: 5,938,769 $\pm 3,407,199$ SD reads) across all bait strengths

374 and Kapa kits tested (Table S2). Quality and adapter trimming lead to the removal of 1.8 to

$37510.5 \%$ reads from each sample, resulting in a mean of 5,486,800 $\pm 2,092,161$ SD trimmed reads

376 per sample (Tables S2, S3). Trimmed reads were assembled into 4,699 to 327,623 contigs per

377 sample (mean: $92,076 \pm 65,772$ SD contigs) with a mean length of $384 \pm 27$ bp (range: 224 to

37832,406 bp) using Trinity (Tables 2 and S3). Coverage averaged 2.5 to 9.9X per contig. No

379 differences in numbers of contigs or reads were evident between libraries prepared using the two

380 different Kapa kits (Hyper Prep or Hyper Plus) at 1/2 bait strength or between the different bait

381 strengths used (1/8, 1/4, 1/2, full) (Fig. S1, Tables 2 and S3). Using Abyss, trimmed reads were

382 assembled into 43,428 to 763,227 contigs per sample with a mean length of only $179 \pm 24 \mathrm{bp}$.

383 Because contig sizes were much smaller from Abyss than those assembled via Trinity, remaining 384 analyses were conducted on the Trinity-assembled data.

A total of 713 UCE loci and 1,061 exon loci (1,774 total loci out of 1,791 targeted loci) were recovered from the assembled contigs. Mean length of UCE contigs was $598 \pm 158 \mathrm{bp}$ (range: 224 to 3,995 bp) and mean length of exon contigs was $593 \pm 156$ bp (range: 224 to 4,500 bp) (Table S2). No differences in numbers of loci were evident between the two different Kapa kits (Hyper Prep or Hyper Plus) at 1/2 bait strength or between the individuals subjected to the four different bait strengths used (Fig. S1, Tables 2 and S3). The number of loci recovered from each species using a Kapa Hyper prep kit with $1 / 2$ bait strength was highly variable, ranging between 172 to 1034 total loci per sample (mean: $638 \pm 222$ loci) (Tables 2 and S3), although

394 recovered from octocorals (mean: $783 \pm 138$ loci, range: 569-1036 loci) compared to hexacorals

395 (mean: $475 \pm 187$ loci, range: 172-786 loci), even after removing the sample with the fewest loci 396 (498 \pm 172 loci).

397 Alignment lengths, locus number and length, and the number of parsimony informative 398 sites varied depending upon percent ( 25 or 50\%) of taxon occupancy per locus and type of 399 taxonomic dataset (Anthozoa+genome+outgroup, Anthozoa, Hexacorallia, Octocorallia) 
400 included in the GBlocks trimmed alignments (Table 3). The average percentage of parsimony

401 informative sites across all alignments was 39\%. For the comparisons within each of three

402 genera (Acropora, Alcyonium, Sinularia), 382 to 426 loci were retained in the $100 \%$ alignment

403 matrices (Table 4). Mean \% variable sites per locus ranged from 4.7 to 30\%, with the most

404 variation found in the Alcyonium dataset and the least found within Acropora. Percent variation

405 per locus ranged from 0 to 55\%, with only one non-polymorphic locus found in the Acropora

406 dataset.

407 Tree topologies were mostly congruent between the $25 \%$ and $50 \%$

408 Anthozoa+genome+outgroup data matrices using all loci and the Bayesian and ML analyses

409 (Figs. 2 and S2). Bootstrap support and posterior probabilities were higher overall in the 25\%

410 Anthozoa+genome+outgroup ML tree (Fig. 2) compared to the 50\% dataset tree (Fig. 2, Fig.

411 S2). By rooting to the outgroup H. magnipapillata, monophyly for the currently established

412 anthozoan subclasses and the hexacoral orders was recovered in all analyses except that the sister

413 relationship of Ceriantharia to the rest of the hexacorals was not supported in the Bayesian

414 analysis of the $25 \%$ dataset. Only a few branches shifted between the ML trees produced with

415 either of the data matrices. Acropora digitifera was sister to A. muricata in the $50 \%$ dataset, but

416 sister to A. millepora in the $25 \%$ dataset. In Octocorallia, both Cornularia pabloi and

417 Erythropodium caribaeorum shifted positions between $25 \%$ and $50 \%$ datasets. These two species

418 and Tubipora musica also changed positions between Bayesian and ML analyses of the 50\%

419 dataset.

420 Lower bootstrap support was found in ML trees created with only the exon loci (Fig.

421 S3C, S3D) or the UCE loci (Fig. S3A, S3B), but tree topologies were congruent with the few

422 exceptions noted above (Fig. S3). Cerianthids were also found to be sister to all other anthozoans

423 in both $25 \%$ and $50 \%$ exon-locus datasets, but sister to hexacorals in the UCE-locus datasets.

424 Zoanthus cf. pulchellus was sister to the actiniarians in the $25 \%$ exon-locus dataset, but sister to a 425 clade containing Actiniaria, Antipatharia, Corallimorpharia, and Scleractinia in all other datasets

426 (Fig S3).

428 Discussion

429 Our results demonstrate the utility of the target-capture enrichment approach for inferring 430 phylogenomic relationships in the class Anthozoa. To date, a few studies based on transcriptomic 
431 data have recovered well-supported phylogenomic relationships within Anthozoa, but these

432 studies were based on only a handful $(\leq 15)$ of taxa (Zapata et al. 2015; Lin et al. 2016, Pratlong

433 et al. 2017) and were limited in scope. In general, phylogenomic studies based on transcriptomic

434 data have provided well-supported and well-resolved phylogenies based on 100s to 1000s of

435 orthologs (Dunn et al. 2008; Kocot et al. 2011; Zapata et al. 2015). However, obtaining these

436 types of sequencing data can be relatively expensive and requires high-quality RNA, two

437 limitations that hinder the transcriptomic-approach for large datasets. In addition, it is often not

438 feasible to obtain RNA from rare taxa or taxa that have not been properly preserved for

439 transcriptomics, such as museum specimens. In our study, we show that a sequence-capture

440 approach for both UCEs and exons can be used to capture genome-wide data in anthozoans. To

441 date, this approach has not been applied to anthozoans or to marine invertebrates more generally

442 (except Hugall et al. 2016). We successfully designed a novel bait set based on existing

443 transcriptomes and genomes, and captured 1,774 loci from a diversity of anthozoans spanning

$444>500$ million years of divergence (Peterson et al. 2004). This target-enrichment approach has the

445 capability to resolve evolutionary relationships at a wide range of divergence levels, from deep

446 (orders, sub-orders) to shallow levels (species). This novel genomic resource can help to advance

447 studies of systematics, divergence-time estimation, and character evolution in the species-rich

448 class Anthozoa.

449

450 In Vitro Test Results

451 The newly designed bait set successfully enriched 713 UCE loci and 1,061 exon loci

452 across a diversity of anthozoans. These loci had an average of 39\% parsimony informative sites,

453 comparable to the arachnid (30\% PI sites, Starrett et al. 2016) UCE dataset, which targeted

$454 \sim 1,000$ loci. The large range of loci recovered per anthozoan species (172 to 1036 loci) was also

455 similar to the arachnid results (170 to 722 loci). We note that the number of loci recovered from

456 octocorals was much higher than what was recovered from hexacorals. This result is perhaps

457 because we added more octocoral-specific baits to the final bait set. And as we added more

458 octocoral-specific baits, we removed baits that were potential paralogs; the majority of these

459 were designed based on the hexacorals. As was done for the hymenopteran UCE bait set

460 (Branstetter et al. 2017), we need to re-design the baitset and include additional octocoral-

461 specific baits and hexacoral-specific baits to increase the success of locus capture. We will also 
462 design separate octocoral- and hexacoral-specific bait sets so that additional loci specific to each

463 sub-class can be targeted. Nevertheless, this first bait design and in vitro results from 33 taxa

464 demonstrate the promising utility of the target-capture method for resolving anthozoan

465 relationships across deep divergence levels.

466 The number of variable sites found at loci recovered from within three genera

467 demonstrates that this is also a promising approach for species-level phylogenetics. Within all

468 three genera examined, variable sites ranged up to 55\% per locus, with a mean variation across

469 all loci of 4.7, 5.5, and 30\% in Sinularia, Acropora, and Alcyonium, respectively. The high

470 variation seen within Alcyonium is consistent with unpublished data (C. McFadden, unpubl. data)

471 that suggest the three species are perhaps different genera. For Sinularia, average divergence

472 estimates are also higher $(\sim 10 \mathrm{X})$ than what has been demonstrated in other studies using

473 mitochondrial barcoding markers (McFadden et al. 2009). In fact, a 0.5\% divergence level at an

474 extended mitochondrial barcode $(m t M u t S+i g r I+C O I)$ was proposed as a conservative criterion

475 for species delimitation (McFadden et al. 2011, 2014). Similarly, low divergence estimates at

476 mitochondrial barcoding markers have been found among hexacoral congeners (Shearer and

477 Coffroth 2008; Brugler et al. 2013; Gonzalez-Muñoz et al. 2015). Thus, these UCE and exon

478 loci are promising for resolving species boundaries, although the level of intraspecific variation

479 has yet to be determined. Our UCE and exon locus datasets may serve as an alternative resource

480 to RADseq to address species-boundary questions while simultaneously allowing for data to be

481 combined and examined across deeper levels.

482 Because this was the first time the target-enrichment UCE approach had been tested on

483 anthozoans, we compared different concentrations of baits and different library preparation kits

484 to determine whether or not particular methods would recover more loci. We found no

485 differences in the number of loci recovered using different concentrations of baits in the

486 hybridization and enrichment protocols. This bait-strength test suggested that the number of

487 hybridizations obtained from one standard reaction could, at least, be doubled. We also found no

488 differences between the two different Kapa kits used. The enzymatic DNA shearing that can be

489 performed with the Kapa Hyper Plus kit may be useful for researchers who do not have access to 490 a sonicator.

491 Following internal trimming with GBlocks and aligning of conserved loci, the mean

492 locus length was much shorter ( 190 bp) compared to the mean length of un-trimmed loci ( $\sim 600$ 
493 bp). Therefore, some of the loci included in the ML analyses were relatively short (<100 bp),

494 particularly in the Anthozoa+genome+outgroup dataset. In alignments between highly divergent

495 taxa (such as between hexacorals and octocorals), numerous poorly aligned positions and

496 divergent positions were filtered with GBlocks. In contrast, the locus size was considerably

497 higher within genera ( $525 \mathrm{bp}$ ) because of fewer poorly aligned and divergent positions. Perhaps

498 re-performing the GBlocks internal trimming with less stringent parameters would increase the

499 size of loci in alignments of divergent taxa. Stringent alignment filtering, as done with GBlocks,

500 can not only increase the proportion of unresolved branches, but can also lead to well-supported

501 branches that are in fact incorrect (Tan et al. 2015). Different methods of aligning and filtering

502 data will be explored in future work.

503 The phylogenies produced from the in vitro data were highly supported despite low

504 overall taxon occupancy (>25 or $50 \%$ matrices) and inclusion of short loci. There were a few

505 nodes that had low support and a few branches that shifted between the different taxon

506 occupancy datasets, particularly in the Octocorallia. In addition to stringent filtering as discussed

507 above, sources of incongruence and low bootstrap support could include compositional bias,

508 saturation, violations of model assumptions (Jeffroy et al. 2006) and/or missing data. Missing

509 data, however, are generally not problematic if there are a reasonable number of informative

510 characters (see Streicher et al. 2015). Rather, incongruence and low support at a few nodes is

511 perhaps due to incomplete taxon sampling (Wiens 2005; Wiens and Tiu 2012). Although a

512 diversity of taxa from across the clades was selected for in vitro analyses, several lineages were

513 not represented, particularly in the Octocorallia. Outgroup choice and taxon evenness can also

514 impact topology and clade support in UCE phylogenomics (Branstetter et al. 2017). Future

515 efforts will need to incorporate more thorough taxon sampling.

516 In general, the inferred phylogenetic relationships corresponded to those found in

517 previous studies (Zapata et al. 2015; Rodríguez et al. 2014), although there were a few

518 exceptions. One exception was the position of the stoloniferan octocoral C. pabloi. In all

519 datasets, this species was nested within the clade containing sea pens (Pennatulacea) and

520 calcaxonians (C. tricaulis, Keratoisidinae sp.), but this species has been previously found to be

521 sister to the rest of the octocorals based on mitochondrial data (McFadden and van Ofwegen

522 2012). The superfamily Actinostoloidea (Sicyonis sp., Stomphia sp.) was recovered as sister to

523 superfamily Actinioidea (Actinostella sp., Isosicyonis alba) in all datasets. This result differed 
524 from the combined mitochondrial and nuclear rDNA dataset of Rodriguez et al. (2014), which

525 instead recovered Actinostoloidea as sister to both Actinioidea and Metridioidea (Lebrunia

526 danae, E. pallida, Bunodeopsis sp.). Furthermore, trees in our study were rooted to $H$.

527 magnipapillata, based on the results of Zapata et al. (2015); however, the unrooted trees

528 indicated that $H$. magnipapillata was sister to the Octocorallia, a relationship (i.e., a paraphyletic

529 Anthozoa) that has been noted by mitochondrial data (Park et al. 2012, Kayal et al. 2013), but

530 not supported by phylogenomic analyses of transcriptomic data (Zapata et al. 2015). Zapata et al.

531 (2015) also found that the position of the order Ceriantharia was phylogenetically unstable.

532 Similarly, we found that the placement of Ceriantharia changed between the different exon and

533 UCE datasets. The topologies resulting from exon data placed the ceriantharians as sister to all

534 remaining anthozoans, a relationship also suggested by analysis of $16 \mathrm{~S}$ and $18 \mathrm{~S}$ ribosomal DNA

535 (Stampar et al. 2014). Trees from UCE loci had ceriantharians as sister to hexacorals; a

536 relationship supported by combined mitochondrial and nuclear rDNA data (Rodríguez et al.

537 2014). Future work must include different outgroup choices (i.e., sponges, bilateria, other

538 cnidarians), while closely examining the distribution and strength of phylogenetic signal. This

539 will help clarify the source of incongruence and resolve which loci strongly influence the

540 resolution of a given 'contentious' branch (Shen et al. 2017).

541 Whether or not scleractinians are monophyletic has been a controversial topic as a result

542 of different phylogenetic analyses. In 2006, Medina et al. reported that scleractinians were

543 polyphyletic with corallimorpharians. The "naked coral hypothesis" was thus proposed,

544 suggesting that corallimorpharians arose from a scleractinian ancestor that had undergone

545 skeletal loss during paleoclimate conditions when the oceans experienced increased $\mathrm{CO}_{2}$

546 concentrations (Medina et al. 2006). Since that study, other studies based on transcriptomic data

547 (Lin et al. 2016), rDNA (Fukami et al. 2008), and mitochondrial data (Fukami et al. 2008; Park

548 et al. 2012; Kayal et al. 2013; Kitahara et al. 2014) recovered a monophyletic Scleractinia with

549 corallimorpharians as the sister clade. Our results also recovered a monophyletic Scleractinia,

550 thus supporting the conclusions of others that corallimorpharians are not naked corals. However,

551 increased sampling of robust, complex, and basal scleractinians is necessary to conclusively

552 address this issue.

553

$554 \quad$ Future Research Directions

This article is protected by copyright. All rights reserved 
The in silico and in vitro tests of the novel bait set demonstrate that the target-enrichment

556 approach of UCEs and exons is a promising new genomic resource for inferring phylogenetic

557 relationships among anthozoans. Using this bait set, target-capture enrichment of the UCE and

558 exon loci from at least 192 additional anthozoans is currently underway to further our

559 understanding of character evolution and systematics of the clade. Adding more taxa will likely

560 increase the accuracy of the phylogenetic inference. We also plan to use additional outgroup

561 taxa, including medusozoan cnidarians and sponges, to help address whether or not octocorals

562 are sister to hexacorals or medusozoans and resolve the position of ceriantharians. Finally, we

563 plan to re-design the bait sets to create hexacoral- and octocoral-specific bait sets. We will

564 include additional baits to increase the capture efficiency of loci that were targeted in this study,

565 while adding more loci that are specific to each sub-class. This target-enrichment approach

566 provides a promising genomic resource to resolve phylogenetic relationships at deep to shallow

567 levels of divergence, considerably advancing the current state of knowledge of anthozoan

568 evolution.

569

570 Acknowledgements

571 Funding was provided by NSF-DEB \#1457817 to CSM and \#1457581 to ER. The 2013 HIMB

572 Pauley program also provided funds to AMQ for preliminary analyses. C. Oliveros and J. Salter

573 helped during lab work and J. Bast helped during preliminary data analyses. F. Zapata and C.

574 Dunn provided transcriptomic datasets. S. Lengyel, E. Cordes, and R. Kulathinal aided DD with

575 the Paramuricea transcriptome assembly, which was funded by a grant from Gulf of Mexico

576 Research to support the "Ecosystem Impacts of Oil and Gas in the Gulf" (ECOGIG) research

577 consortium. E. Bush provided computing support. Specimens from Irish waters were collected

578 during cruise CE14004 on the RV Celtic Explorer led by L. Allcock, NUI Galway, and

579 supported by the Marine Institute's Shiptime Programme. Special thanks to Z. Forsman, R.

580 Toonen, and I. Knapp for organizing the 2013 HIMB Pauley program. Thanks to J. Ryan and

581 three anonymous reviewers for constructive comments and suggestions.

582

583 Author Contributions

584 
585 AMQ, CSM, ER, and BCF conceived and designed this study. AMQ designed the baits, 586 conducted library preparation, target enrichment, and data analyses, and wrote the initial draft of 587 the manuscript with significant contributions from CSM. BCF developed protocols and guided 588 AMQ in laboratory and bioinformatic analyses. LFD helped with preliminary analyses. MB, ER, 589 and CSM extracted DNA. ICB, DMD, SF, SH, SL, DJM, CP, GRB, CRP, and JAS provided 590 genomic or transcriptomic data for analysis. TB provided samples. All authors edited and 591 approved the final version of this manuscript.

\section{Data Accessibility}

595 Tree and alignment files: Data Dryad Entry http://dx.doi.org/10.5061/dryad.36n40

596 Raw Data: SRA Genbank SUB3122367, BioSample \#SAMN07774920-4952

597 Anthozoan bait set: Data Dryad Entry http://dx.doi.org/10.5061/dryad.36n40

\section{References}

600

601 Aberer, A. J., Kobert, K., \& Stamatakis, A. (2014). ExaBayes: massively parallel Bayesian tree 602 inference for the whole-genome era. Molecular Biology and Evolution, 31(10), 2553-2556.

603

604 Althoff, D. M., Gitzendanner, M. A., \& Segraves, K. A. (2007). The utility of amplified 605 fragment length polymorphisms in phylogenetics: a comparison of homology within and 606 between genomes. Systematic Biology, 56(3), 477-484.

608 Baca, S. M., Alexander, A., Gustafson, G. T., \& Short, A. E. (2017). Ultraconserved elements 609 show utility in phylogenetic inference of Adephaga (Coleoptera) and suggest paraphyly of 610 'Hydradephega'. Systematic Entomology, doi:10.1111/syen.12244

612 Berntson, E. A., Bayer, F. M., McArthur, A. G., \& France, S. C. (2001). Phylogenetic 613 relationships within the Octocorallia (Cnidaria: Anthozoa) based on nuclear 18S rRNA 614 sequences. Marine Biology, 138(2), 235-246. 
616 Bi, K., Vanderpool, D., Singhal, S., Linderoth, T., Moritz, C., \& Good, J. M. (2012).

617 Transcriptome-based exon capture enables highly cost-effective comparative genomic data

618 collection at moderate evolutionary scales. BMC Genomics, 13(1), 403.

620 Bilewitch, J.P., \& Degnan, S. M. (2011). A unique horizontal gene transfer event has provided 621 the octocoral mitochondrial genome with an active mismatch repair gene that has potential for an 622 unusual self-contained function. BMC Evolutionary Biology, 11(1), 228.

624 Branstetter, M. G., Longino, J. T., Ward, P. S., \& Faircloth, B. C. (2017). Enriching the ant tree 625 of life: enhanced UCE bait set for genome- scale phylogenetics of ants and other

626 Hymenoptera. Methods in Ecology and Evolution. 8: 768-776. doi:10.1111/2041-210X.12742

628 Brugler, M. R., Opresko, D. M., \& France, S. C. (2013). The evolutionary history of the order 629 Antipatharia (Cnidaria: Anthozoa: Hexacorallia) as inferred from mitochondrial and nuclear 630 DNA: implications for black coral taxonomy and systematics. Zoological Journal of the Linnean 631 Society, 169(2),312-361.

633 Bushnell, B. (2015). BBMap short-read aligner, and other bioinformatics tools. Available from: 634 sourceforge.net/projects/bbmap.

636 Castresana, J. (2000). Selection of conserved blocks from multiple alignments for their use in 637 phylogenetic analysis. Molecular Biology and Evolution, 17(4), 540-552.

639 Combosch, D. J., \& Vollmer, S. V. (2015). Trans-Pacific RAD-Seq population genomics 640 confirms introgressive hybridization in Eastern Pacific Pocillopora corals. Molecular 641 Phylogenetics and Evolution, 88, 154-162.

643 Crawford, N. G., Faircloth, B. C., McCormack, J. E., Brumfield, R. T., Winker, K., \& Glenn, T. 644 C. (2012). More than 1000 ultraconserved elements provide evidence that turtles are the sister 645 group of archosaurs. Biology Letters, 8(5), 783-786. 
647 Daly, M., Fautin, D. G., \& Cappola, V. A. (2003). Systematics of the hexacorallia (Cnidaria:

648 Anthozoa). Zoological Journal of the Linnean Society,139(3), 419-437.

649

650 Daly, M., Brugler, M. R., Cartwright, P., Collins, A. G., Dawson, M. N., Fautin, D. G., ... \&

651 Romano, S. L. (2007). The phylum Cnidaria: a review of phylogenetic patterns and diversity 300

652 years after Linnaeus. Zootaxa, 1668, 127-182.

653

654 Daly, M., Gusmão, L., Reft, A., \& Rodríguez, E. (2010) Phylogenetic signal in mitochondrial 655 and nuclear markers in sea anemones (Cnidaria, Actiniaria). Integrative Comparative Biology $65650(3), 371-388$.

657

658 Daly, M., Crowley, L. M., Larson, P., Rodríguez, E., Saucier, E. H., \& Fautin, D. G. (2017).

659 Anthopleura and the phylogeny of Actinioidea (Cnidaria: Anthozoa: Actiniaria). Organisms

660 Diversity \& Evolution, 1-20.

661

662 Dueñas, L. F., Alderslade, P., \& Sánchez, J. A. (2014). Molecular systematics of the deep-sea 663 bamboo corals (Octocorallia: Isididae: Keratoisidinae) from New Zealand with descriptions of 664 two new species of Keratoisis. Molecular Phylogenetics and Evolution, 74, 15-28.

665

666 Dunn, C. W., Hejnol, A., Matus, D. Q., Pang, K., Browne, W. E., Smith, S. A., ... \& Sørensen, 667 M. V. (2008). Broad phylogenomic sampling improves resolution of the animal tree of 668 life. Nature, 452(7188), 745-749.

669

670 Faircloth, B. C., McCormack, J. E., Crawford, N. G., Harvey, M. G., Brumfield, R. T., \& Glenn,

671 T. C. (2012). Ultraconserved elements anchor thousands of genetic markers spanning multiple

672 evolutionary timescales. Systematic Biology, 61(5), 717-726.

673

674 Faircloth, B. C., Sorenson, L., Santini, F., \& Alfaro, M. E. (2013). A phylogenomic perspective 675 on the radiation of ray-finned fishes based upon targeted sequencing of ultraconserved elements 676 (UCEs). PLoS One, 8(6), e65923, doi:10.1111/2041-210X.12754. 
678 Faircloth, B.C. (2016). PHYLUCE is a software package for the analysis of conserved genomic 679 loci. Bioinformatics, 32(5), 786-788.

680

681 Faircloth, B.C. (2017). Identifying conserved genomic elements and designing universal bait sets 682 to enrich them. Methods in Ecology and Evolution. doi: 10.1111/2041-210X.12754

683

684 Fautin, D.G., \& Mariscal, R.N. (1991). Cnidaria: Anthozoa (Vol. 2, pp. 267-358). New York:

685 Wiley-Liss.

686

687

Forsman, Z.H., Barshis, D.J., Hunter, C.L. and Toonen, R.J., 2009. Shape-shifting corals:

688 molecular markers show morphology is evolutionarily plastic in Porites. BMC Evolutionary

689 Biology, 9(1), p.45.

690

691 Fukami, H., Chen, C. A., Budd, A. F., Collins, A., Wallace, C., Chuang, Y. Y., ... \& Knowlton, 692 N. (2008). Mitochondrial and nuclear genes suggest that stony corals are monophyletic but most 693 families of stony corals are not (Order Scleractinia, Class Anthozoa, Phylum Cnidaria). PLoS 694 One, 3(9), e3222.

695

696 Glenn, T. C., Nilsen, R., Kieran, T. J., Finger, J. W., Pierson, T. W., Bentley, K. E., ... \& Reed, 697 K. (2016). Adapterama I: universal stubs and primers for thousands of dual-indexed Illumina 698 libraries (iTru \& iNext). BioRxiv, 049114.

699

700 González-Muñoz, R., Simões, N., Mascaró, M., Tello-Musi, J. L., Brugler, M. R., \& Rodríguez, 701 E. (2015). Morphological and molecular variability of the sea anemone Phymanthus crucifer

702 (Cnidaria, Anthozoa, Actiniaria, Actinoidea). Journal of the Marine Biological Association of the 703 United Kingdom, 95(1), 69-79.

704

705 Grajales, A., \& Rodríguez, E. (2016). Elucidating diversity within the Aiptasiidae, a widespread

706 cnidarian-dinoflagellate model system (Cnidaria: Anthozoa: Actiniaria: Metridioidea). Molecular

707 Phylogenetics and Evolution, 94(A), 252-263. 
Haas, B. J., Papanicolaou, A., Yassour, M., Grabherr, M., Blood, P. D., Bowden, J., ... \& Regev,

710 A. (2013). De novo transcript sequence reconstruction from RNA-seq using the Trinity platform

711 for reference generation and analysis. Nature Protocols, 8(8), 1494-1512.

713 Hebert, P. D., Ratnasingham, S., \& de Waard, J. R. (2003). Barcoding animal life: cytochrome c 714 oxidase subunit 1 divergences among closely related species. Proceedings of the Royal Society 715 of London B: Biological Sciences, 270(Suppl. 1), S96-S99.

717 Hellberg, M. E. (2006). No variation and low synonymous substitution rates in coral mtDNA 718 despite high nuclear variation. BMC Evolutionary Biology, 6(1), 24.

Herrera, S., \& Shank, T. M. (2016). RAD sequencing enables unprecedented phylogenetic resolution and objective species delimitation in recalcitrant divergent taxa. Molecular

722 Phylogenetics and Evolution, 100, 70-79.

724 Huang, D., Meier, R., Todd, P. A., \& Chou, L. M. (2008). Slow mitochondrial COI sequence 725 evolution at the base of the metazoan tree and its implications for DNA barcoding. Journal of 726 Molecular Evolution, 66(2), 167-174.

Huang, W., Li, L., Myers, J. R., \& Marth, G. T. (2012). ART: a next-generation sequencing read 730 simulator. Bioinformatics, 28(4), 593-594.

Hugall, A. F., O’Hara, T. D., Hunjan, S., Nilsen, R., \& Moussalli, A. (2016). An exon-capture 733 system for the entire class Ophiuroidea. Molecular Biology and Evolution, 33(1), 281-294.

734 Ilves, K. L., \& López- Fernández, H. (2014). A targeted next- generation sequencing toolkit for 735 exon-based cichlid phylogenomics. Molecular Ecology Resources, 14(4), 802-811.

737 Jeffroy, O., Brinkmann, H., Delsuc, F., \& Philippe, H. (2006). Phylogenomics: the beginning of 738 incongruence?. TRENDS in Genetics, 22(4), 225-231. 
740 Johnston, E.C., Z.H. Forsman, J. François Flot, S. Schmidt-Roach, J.H. Pinzón, I.S.S. Knapp, 741 and R.J. Toonen. (2017). A genomic glance through the fog of plasticity and diversification in 742 Pocillopora. Scientific Reports 7 ,

744 Kayal, E., Roure, B., Philippe, H., Collins, A. G., \& Lavrov, D. V. (2013). Cnidarian

745 phylogenetic relationships as revealed by mitogenomics. BMC Evolutionary Biology, 13(1), 5.

747 Katoh, K., Misawa, K., Kuma, K. I., \& Miyata, T. (2002). MAFFT: a novel method for rapid 748 multiple sequence alignment based on fast Fourier transform. Nucleic Acids Research, 30(14),

749 3059-3066.

750

751

Kent, W. J. (2002). BLAT—-the BLAST-like alignment tool. Genome Research,12(4), 656-664.

752

753 Kitahara, M. V., Lin, M. F., Forêt, S., Huttley, G., Miller, D. J., \& Chen, C. A. (2014). The

754 "naked coral” hypothesis revisited-evidence for and against scleractinian monophyly. PLoS

755 One, 9(4), e94774.

756

757 Kocot, K. M., Cannon, J. T., Todt, C., Citarella, M. R., Kohn, A. B., Meyer, A., ... \& Halanych, 758 K. M. (2011). Phylogenomics reveals deep molluscan relationships. Nature, 477(7365), 452-456.

760 Li, H., Handsaker, B., Wysoker, A., Fennell, T., Ruan, J., Homer, N., ... \& Durbin, R. (2009).

761 The sequence alignment/map format and SAMtools. Bioinformatics, 25(16), 2078-2079.

763 Lin, M. F., Chou, W. H., Kitahara, M. V., Chen, C. L. A., Miller, D. J., \& Forêt, S. (2016).

764 Corallimorpharians are not "naked corals": insights into relationships between Scleractinia and

765 Corallimorpharia from phylogenomic analyses. PeerJ, 4, e2463.

767 Lunter, G., \& Goodson, M. (2011). Stampy: a statistical algorithm for sensitive and fast mapping 768 of Illumina sequence reads. Genome Research, 21(6), 936-939. 
770 Manthey, J. D., Campillo, L. C., Burns, K. J., \& Moyle, R. G. (2016). Comparison of target-

771 capture and restriction-site associated DNA sequencing for phylogenomics: a test in cardinalid

772 tanagers (Aves, Genus: Piranga). Systematic Biology, 65(4), 640-650.

773

774 McCormack, J. E., Faircloth, B. C., Crawford, N. G., Gowaty, P. A., Brumfield, R. T., \& Glenn, 775 T. C. (2012). Ultraconserved elements are novel phylogenomic markers that resolve placental 776 mammal phylogeny when combined with species-tree analysis. Genome Research, 22(4), 746777754.

778

779

McCormack, J. E., Hird, S. M., Zellmer, A. J., Carstens, B. C., \& Brumfield, R. T. (2013a).

780 Applications of next-generation sequencing to phylogeography and phylogenetics. Molecular

781 Phylogenetics and Evolution, 66(2), 526-538.

782

783 McCormack, J. E., Harvey, M. G., Faircloth, B. C., Crawford, N. G., Glenn, T. C., \& Brumfield, 784 R. T. (2013b). A phylogeny of birds based on over 1,500 loci collected by target enrichment and 785 high-throughput sequencing. PLoS One,8(1), e54848.

786

787

McCormack, J.E., Tsai, W. L., \& Faircloth, B. C. (2016). Sequence capture of ultraconserved 788 elements from bird museum specimens. Molecular Ecology Resources, 16(5), 1189-1203.

McFadden, C. S., Alderslade, P., Van Ofwegen, L. P., Johnsen, H., \& Rusmevichientong, A.

791 (2006). Phylogenetic relationships within the tropical soft coral genera Sarcophyton and

792 Lobophytum (Anthozoa, Octocorallia). Invertebrate Biology, 125(4), 288-305.

793

794 McFadden, C. S., Van Ofwegen, L. P., Beckman, E. J., Benayahu, Y., \& Alderslade, P. (2009).

795 Molecular systematics of the speciose Indo- Pacific soft coral genus, Sinularia (Anthozoa:

796 Octocorallia). Invertebrate Biology, 128(4), 303-323.

798 McFadden, C. S., Sánchez, J. A., \& France, S. C. (2010). Molecular phylogenetic insights into

799 the evolution of Octocorallia: a review. Integrative and Comparative Biology, 50, 389-410. 
801 McFadden, C. S., Benayahu, Y., Pante, E., Thoma, J. N., Nevarez, P. A., \& France, S. C. (2011).

802 Limitations of mitochondrial gene barcoding in Octocorallia. Molecular Ecology

803 Resources, 11(1), 19-31.

804

805 McFadden, C. S., \& van Ofwegen, L. P. (2012). Stoloniferous octocorals (Anthozoa,

806 Octocorallia) from South Africa, with descriptions of a new family of Alcyonacea, a new genus

807 of Clavulariidae, and a new species of Cornularia (Cornulariidae). Invertebrate

808 Systematics, 26(4), 331-356.

809

810 McFadden, C. S., Brown, A. S., Brayton, C., Hunt, C. B., \& van Ofwegen, L. P. (2014).

811 Application of DNA barcoding in biodiversity studies of shallow-water octocorals: molecular

812 proxies agree with morphological estimates of species richness in Palau. Coral Reefs, 33(2), 275-

813286.

814

815 McFadden, C. S., Haverkort-Yeh, R., Reynolds, A. M., Halàsz, A., Quattrini, A. M., Forsman, Z.

816 H., Benayahu, Y., \& Toonen, R. J. (2017). Species boundaries in the absence of morphological,

817 ecological or geographical differentiation in the Red Sea octocoral genus Ovabunda

818 (Alcyonacea: Xeniidae). Molecular Phylogenetics and Evolution, 112, 174-184.

819

820 Medina, M., Weil, E., \& Szmant, A. M. (1999). Examination of the Montastraea annularis

821 species complex (Cnidaria: Scleractinia) using ITS and COI sequences. Marine

822 Biotechnology, 1(1), 89-97.

823

824 Medina, M., Collins, A. G., Takaoka, T. L., Kuehl, J. V., \& Boore, J. L. (2006). Naked corals:

825 skeleton loss in Scleractinia. Proceedings of the National Academy of Sciences, 103(24), 9096-

8269100.

827

828 Pante, E., Abdelkrim, J., Viricel, A., Gey, D., France, S. C., Boisselier, M. C., \& Samadi, S.

829 (2015). Use of RAD sequencing for delimiting species. Heredity, 114(5), 450-459.

830

This article is protected by copyright. All rights reserved 
831 Park, E., Hwang, D. S., Lee, J. S., Song, J. I., Seo, T. K., \& Won, Y. J. (2012). Estimation of

832 divergence times in cnidarian evolution based on mitochondrial protein-coding genes and the

833 fossil record. Molecular Phylogenetics and Evolution, 62(1), 329-345.

834

835 Peterson, K.J., Lyons, J.B., Nowak, K.S., Takacs, C.M., Wargo, M.J. \& McPeek, M.A. (2004).

836 Estimating metazoan divergence times with a molecular clock. Proceedings of the National

837 Academy of Sciences, 101(17), 6536-6541.

838

839

Pinzon, J. H., \& LaJeunesse, T.D. (2011). Species delimitation of common reef corals in the

840 genus Pocillopora using nucleotide sequence phylogenies, population genetics and symbiosis

841 ecology. Molecular Ecology, 20(2), 311-325.

842

843 Prada, C., DeBiasse, M. B., Neigel, J. E., Yednock, B., Stake, J. L., Forsman, Z. H., ... \&

844 Hellberg, M. E. (2014). Genetic species delineation among branching Caribbean Porites

845 corals. Coral Reefs, 33(4), 1019-1030.

846

847 Pratlong, M., Rancurel, C., Pontarotti, P., \& Aurelle, D. (2017). Monophyly of Anthozoa

848 (Cnidaria): why do nuclear and mitochondrial phylogenies disagree? Zoologica Scripta, 46(3), $849 \quad 363-371$.

850

851 Quinlan, A. R., \& Hall, I. M. (2010). BEDTools: a flexible suite of utilities for comparing

852 genomic features. Bioinformatics, 26(6), 841-842.

853

854 Rodríguez,,E., Barbeitos, M. S., Brugler, M. R., Crowley, L. M., Grajales, A., Gusmão, L., ... \&

855 Daly, M. (2014). Hidden among sea anemones: the first comprehensive phylogenetic

856 reconstruction of the order Actiniaria (Cnidaria, Anthozoa, Hexacorallia) reveals a novel group

857 of hexacorals. PLoS One, 9(5), e96998.

858

859 Rohland, N., \& Reich, D. (2012). Cost-effective, high-throughput DNA sequencing libraries for 860 multiplexed target capture. Genome research, 22(5), 939-946.

861 
862 Ruane, S., \& Austin, C. C. (2017). Phylogenomics using formalin- fixed and 100+ year- old

863 intractable natural history specimens. Molecular Ecology Resources, doi:10.1111/1755-

$864 \quad 0998.12655$

865

866 Ryu, T., Seridi, L., \& Ravasi, T. (2012). The evolution of ultraconserved elements with different

867 phylogenetic origins. BMC Evolutionary Biology, 12(1), 236.

868

869 Shearer, T. L., Van Oppen, M. J. H., Romano, S. L., \& Wörheide, G. (2002). Slow mitochondrial 870 DNA sequence evolution in the Anthozoa (Cnidaria). Molecular Ecology, 11(12), 2475-2487.

871

872 Shearer, T. L., \& Coffroth, M. A. (2008). DNA BARCODING: Barcoding corals: limited by 873 interspecific divergence, not intraspecific variation. Molecular Ecology Resources, 8(2), 247874255.

875

876 Shen, X. X., Hittinger, C. T., \& Rokas, A. (2017). Contentious relationships in phylogenomic 877 studies can be driven by a handful of genes. Nature Ecology \& Evolution, 1, 0126.

878

879

880

Simpson, J. T., Wong, K., Jackman, S. D., Schein, J. E., Jones, S. J., \& Birol, I. (2009). ABySS:

881

882 Smit, AFA, Hubley, R \& Green, P. (2015). RepeatMasker Open-4.0.

$883<$ http://www.repeatmasker.org>.

884

885 Smith, B. T., Harvey, M. G., Faircloth, B. C., Glenn, T. C., \& Brumfield, R. T. (2014). Target

886 capture and massively parallel sequencing of ultraconserved elements for comparative studies at 887 shallow evolutionary time scales. Systematic Biology, 63(1), 83-95.

888

889 Stamatakis, A. (2014). RAxML version 8: a tool for phylogenetic analysis and post-analysis of 890 large phylogenies. Bioinformatics, 30(9), 1312-1313.

891 
892 Stampar, S. N., Maronna, M. M., Kitahara, M. V., Reimer, J. D., \& Morandini, A. C. (2014).

893 Fast-evolving mitochondrial DNA in Ceriantharia: a reflection of hexacorallia paraphyly? PLoS

894 One, 9(1), e86612.

895

896 Starrett, J., Derkarabetian, S., Hedin, M., Bryson, R. W., McCormack, J. E., \& Faircloth, B. C.

897 (2016). High phylogenetic utility of an ultraconserved element probe set designed for

898 Arachnida. Molecular Ecology Resources, 17(4), 812-823, doi:10.1111/1755-0998.12621

899

900 Streicher, J. W., Schulte, J. A., \& Wiens, J. J. (2015). How should genes and taxa be sampled for

901 phylogenomic analyses with missing data? An empirical study in iguanian lizards. Systematic

902 Biology, 65(1), 128-145.

903

904 Talavera, G., \& Castresana, J. (2007). Improvement of phylogenies after removing divergent and

905 ambiguously aligned blocks from protein sequence alignments. Systematic Biology, 56(4), 564-

906577.

907

908 Tan, G., Muffato, M., Ledergerber, C., Herrero, J., Goldman, N., Gil, M., \& Dessimoz, C.

909 (2015). Current methods for automated filtering of multiple sequence alignments frequently

910 worsen single-gene phylogenetic inference. Systematic Biology, 64(5), 778-791.

911

912 Van Oppen, M. J. H., Willis, B. L., Van Vugt, H. W. J. A., \& Miller, D. J. (2000). Examination

913 of species boundaries in the Acropora cervicornis group (Scleractinia, Cnidaria) using nuclear

914 DNA sequence analyses. Molecular Ecology, 9(9), 1363-1373.

915

916 Vollmer, S. V., \& Palumbi, S. R. (2004). Testing the utility of internally transcribed spacer

917 sequences in coral phylogenetics. Molecular Ecology,13(9), 2763-2772.

918

919 Wiens, J. J. (2005). Can incomplete taxa rescue phylogenetic analyses from long-branch

920 attraction? Systematic Biology, 54(5), 731-742.

921 
922 Wiens, J. J., \& Tiu, J. (2012). Highly incomplete taxa can rescue phylogenetic analyses from the 923 negative impacts of limited taxon sampling. PLoS One, 7(8), e42925.

924

925 Zapata, F., Goetz, F. E., Smith, S. A., Howison, M., Siebert, S., Church, S. H., ... \& Daly, M.

926 (2015). Phylogenomic analyses support traditional relationships within Cnidaria. PLoS

927 One, 10(10), e0139068

928

929

930

931

932 Figure Captions

933

934 Figure 1. Maximum likelihood phylogenies from in silico analyses. A) Phylogeny constructed

935 with a 138,778 bp concatenated genomic dataset (522 loci) and rooted to Hydra magnipapillata.

936 B) Phylogeny constructed with 220,139 bp concatenated transcriptome dataset (407 loci) with

937 the Hexacorallia rooted to the Octocorallia. Bootstrap support (b.s.) values are followed by

938 posterior probabilities (p.p.) from Bayesian analyses. * $=100 \%$ b.s. and 1.0 p.p. Branches are

939 color coded by order (green=Ceriantharia, pink=Zoantharia, purple=Scleractinia,

940 blue=Actiniaria, red=Alcyonacea, grey=Pennatulacea)

941

942 Figure 2. Maximum likelihood phylogeny on the Anthozoa+genome+outgroup 25\% matrix

$943(257,728 \mathrm{bp}, 1378$ loci). The tree includes 33 taxa from the in vitro test, 9 genome-enabled taxa,

944 and the outgroup Hydra magnipapillata. Bootstrap support (b.s.) values are followed by

945 posterior probabilities (p.p) from Bayesian analyses *=100\% b.s. and 1.0 p.p.; - =not supported

946 by Bayesian analysis. Branches are color coded by order (green=Ceriantharia, pink=Zoantharia,

947 brown=Antipatharia, purple=Scleractinia, lt. blue=Corallimorpharia, blue=Actiniaria,

948 red=Alcyonacea grey=Pennatulacea) 


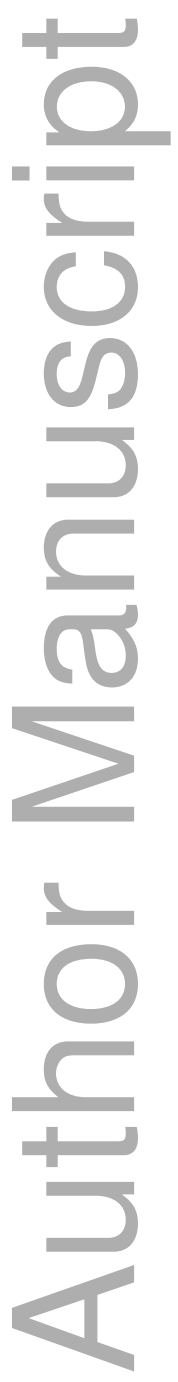

This article is protected by copyright. All rights reserved 
Table 1. Number of loci recovered from in silico analyses after initial and final screens for potential paralogs. Also included are the N50 and number of scaffolds for each genome/transcriptome used in analyses.

\begin{tabular}{|c|c|c|c|c|c|c|}
\hline () & & & & & \multicolumn{2}{|c|}{ \# Loci Recovered } \\
\hline Sub-class & Order & Species & \#Scaffolds & N50 & Initial Screen & Final Screen \\
\hline \multicolumn{7}{|l|}{ Genomes } \\
\hline Hexacorallia & Actiniaria & Exaiptasia pallida & 4,312 & 442,145 & 518 & 417 \\
\hline Hexacorallia & Actiniaria & Nematostella vectensis & 10,804 & 472,588 & 496 & 421 \\
\hline Hexacorallia & Actiniaria & Stomphia sp. & 479,824 & 948 & 44 & 25 \\
\hline Hexacorallia & Scleractinia & Acropora digitifera & 4,765 & 191,489 & 462 & 395 \\
\hline Hexacorallia & Scleractinia & Acropora millepora & 12,559 & 181,771 & 511 & 414 \\
\hline Octocorallia & Alcyonacea & Antillogorgia bipinnata & 426,978 & 3,212 & 230 & 134 \\
\hline Octocorallia & Alcyonacea & Pacifigorgia irene & 183,211 & 2,323 & 547 & 491 \\
\hline Octocorallia & Alcyonacea & Paragorgia stephencairnsi & 700,190 & 1,793 & 453 & 371 \\
\hline Octocorallia & Pennatulacea & Renilla muelleri & 4,114 & 19,024 & 599 & 529 \\
\hline Hydrozoa & Aplanulata & Hydra magnipapillata & 126,667 & 10,113 & 449 & 99 \\
\hline \multicolumn{7}{|l|}{ Transcriptomes } \\
\hline Hexacorallia & Actiniaria & Anemonia sp. & 14,279 & 703 & 235 & 106 \\
\hline Hexacorallia & Actiniaria & Anthopleura elegantissima & 142,934 & 1,489 & 364 & 207 \\
\hline Hexacorallia & Actiniaria & Edwardsia lineata & 90,440 & 1,035 & 841 & 623 \\
\hline Hexacorallia & Actiniaria & Exaiptasia pallida & 60,101 & 2,159 & 553 & 264 \\
\hline
\end{tabular}




\begin{tabular}{lllllll} 
Hexacorallia & Actiniaria & Metridium sp. & 10,885 & 752 & 222 & 111 \\
Hexacorallia & Actiniaria & Nematostella vectensis & 27,273 & 1,524 & 836 & 614 \\
Hexacorallia & Ceriantharia & Cerianthidae sp. & 12,074 & 646 & 336 & 157 \\
Hexacorallia & Scleractinia & Acropora digitifera & 36,780 & 1,575 & 857 & 620 \\
Hexacorallia & Scleractinia & Acropora hyacinthus & 67,844 & 422 & 392 & 296 \\
Hexacorallia & Scleractinia & Fungia scutaria & 155,914 & 1,619 & 290 & 188 \\
Hexacorallia & Scleractinia & Montastraea cavernosa & 200,222 & 2,145 & 206 & 128 \\
Hexacorallia & Scleractinia & Orbicella faveolata & 32,463 & 1,736 & 408 & 194 \\
Hexacorallia & Scleractinia & Pocillopora damicornis & 70,786 & 976 & 242 & 152 \\
Hexacorallia & Scleractinia & Porites astreoides & 30,740 & 661 & 379 & 243 \\
Hexacorallia & Scleractinia & Platygyra daedalea & 51,200 & 684 & 483 & 284 \\
Hexacorallia & Zoantharia & Protopalythoa variabilis & 130,118 & 1,187 & 521 & 204 \\
Octocorallia & Alcyonacea & Anthomastus sp. & 9,368 & 610 & 339 & 272 \\
Octocorallia & Alcyonacea & Corallium rubrum & 48,074 & 2,470 & 734 & 606 \\
Octocorallia & Alcyonacea & Eunicea flexuosa & 165,709 & 1,095 & 580 & 507 \\
Octocorallia & Alcyonacea & Gorgonia ventalina & 90,230 & 1,149 & 731 & 670 \\
Octocorallia & Alcyonacea & Keratoisidinae & 12,385 & 702 & 541 & 429 \\
Octocorallia & Alcyonacea & Nephthyigorgia sp. & 14,677 & 762 & 698 & 619 \\
Octocorallia & Alcyonacea & Paramuricea sp. & 25,189 & 2,645 & 834 & 747 \\
Octocorallia & Alcyonacea & Scleronephthya sp. & 8,401 & 683 & 313 & 257 \\
\hline
\end{tabular}


Table 2. List of species used in the in vitro test of designed baits with assembly summary statistics. Results are from the Kapa Hyper Prep and Hyper Plus (in bold) library preparation kits with target enrichments performed using $250 \mathrm{ng}$ of baits.

\begin{tabular}{|c|c|c|c|c|c|c|}
\hline 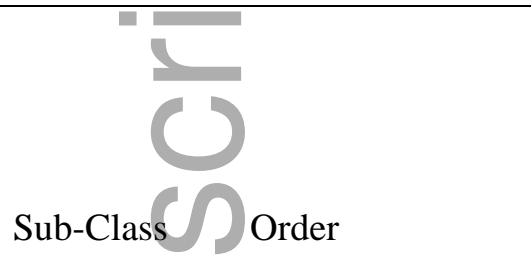 & Species & \# Contigs & $\begin{array}{l}\text { Mean } \\
\text { Contig } \\
\text { Length } \\
\text { (bp) }\end{array}$ & \# UCEs & $\begin{array}{l}\text { \# Exon } \\
\text { Loci }\end{array}$ & $\begin{array}{l}\text { Total \# } \\
\text { Loci }\end{array}$ \\
\hline Hexacorallia Actiniaria & Actinostella sp. & 184,605 & 440 & 345 & 441 & 786 \\
\hline Hexacorallia Actiniaria & Bunodeopsis sp. & 82,100 & 413 & 285 & 257 & 364 \\
\hline Hexacorallia Actiniaria & Halcurias pilatus*^ & $89,449 / 27,355$ & $379 / 387$ & $254 / 158$ & $258 / 144$ & $512 / 302$ \\
\hline Hexacorallia Actiniaria & Isosicyonis alba*^ & $88,159 / 37,119$ & $368 / 360$ & $210 / 146$ & $184 / \mathbf{1 3 8}$ & $394 / 284$ \\
\hline Hexacorallia Actiniaria & Lebrunia danae & 187,114 & 403 & 340 & 368 & 708 \\
\hline Hexacorallia Actiniaria & Sicyonis sp. ${ }^{\wedge *}$ & $50,490 / \mathbf{1 0 5 , 3 2 6}$ & $402 / 407$ & $174 / 238$ & $287 / 249$ & $461 / 487$ \\
\hline Hexacorallia Antipatharia & Antipathes grandis ${ }^{\wedge} \%$ & $\mathbf{5 7 , 9 5 0}$ & 323 & 185 & 197 & 382 \\
\hline Hexacorallia Antipatharia & Myriopathes ulex $\wedge^{\wedge}$ & 96,476 & 356 & 248 & 267 & 515 \\
\hline Hexacorallia Ceriantharia & Cerianthus membranaceus ${ }^{\wedge *}$ & $146,327 / \mathbf{1 4 3 , 2 2 1}$ & $397 / 372$ & $206 / 212$ & $231 / 227$ & $437 / 439$ \\
\hline Hexacorallia Ceriantharia & Pachycerianthus sp. & 101,786 & 426 & 188 & 198 & 386 \\
\hline Hexacorallia Corallimorpharia & Corynactis chilensis ${ }^{\wedge *}$ & 15,433/44,166 & 362 & $95 / 179$ & $77 / \mathbf{1 8 7}$ & $172 / 366$ \\
\hline Hexacorallia Corallimorpharia & Discosoma carlgreni & 37,499 & 353 & 223 & 260 & 483 \\
\hline Hexacorallia Scleractinia & Acropora muricata & 93,433 & 378 & 322 & 408 & 730 \\
\hline Hexacorallia Scleractinia & Pavona sp.^ ${ }^{\%}$ & 57,223 & 340 & 232 & 251 & 483 \\
\hline Hexacorallia Scleractinia & Pocillipora damicornis & 4,699 & 339 & 123 & 105 & 228 \\
\hline
\end{tabular}

This article is protected by copyright. All rights reserved 


\begin{tabular}{lllllll} 
Hexacorallia Scleractinia & Stylophora pistillata & 162,597 & 394 & 297 & 311 & 606 \\
Hexacorallia Zoantharia & Zoanthus cf. pulchellus & 164,870 & 373 & 209 & 195 & 542 \\
Octocorallia Alcyonacea & Alcyonium acaule & 93,846 & 401 & 363 & 543 & 906 \\
Octocorallia Alcyonacea & Alcyonium digitatum & 43,531 & 393 & 343 & 486 & 829 \\
Octocorallia Alcyonacea & Alcyonium haddoni & 66,764 & 414 & 348 & 570 & 918 \\
Octocorallia Alcyonacea & Chrysogorgia tricaulis & 111,571 & 413 & 235 & 331 & 566 \\
Octocorallia Alcyonacea & Clavularia inflata & 84,673 & 352 & 247 & 325 & 572 \\
Octocorallia Alcyonacea & Coelogorgia palmosa & 127,823 & 437 & 367 & 572 & 939 \\
Octocorallia Alcyonacea & Cornularia pabloi & 107,331 & 371 & 292 & 359 & 651 \\
Octocorallia Alcyonacea & Erythropodium caribaeorum & 119,210 & 398 & 316 & 417 & 733 \\
Octocorallia Alcyonacea & Keratoisidinae sp. & 70,544 & 426 & 233 & 344 & 577 \\
Octocorallia Alcyonacea & Parasphaerasclera valdiviae & 85,199 & 404 & 323 & 443 & 766 \\
Octocorallia Alcyonacea & Plexaura kuna & 105,208 & 393 & 423 & 611 & 1034 \\
Octocorallia Alcyonacea & Sinularia slieringsi & 75,970 & 377 & 321 & 516 & 837 \\
Octocorallia Alcyonacea & Sinularia lochmodes & 58,759 & 386 & 314 & 514 & 828 \\
Octocorallia Alcyonacea & Sinularia maxima & 42,099 & 366 & 304 & 528 & 832 \\
Octocorallia Alcyonacea & Tubipora musica & 44,753 & 369 & 282 & 451 & 733 \\
Octocorallia Pennatulacea & Virgularia schultzei & 49,954 & 381 & 269 & 509 & 777 \\
\hline Kapa & & &
\end{tabular}

\footnotetext{
$\wedge$ Kapa HyperPlus Kit Trial

\% Kapa Hyper Prep Kit Trial Library failed

* Probe Concentration Trials
}

This article is protected by copyright. All rights reserved 
Table 3. Allignment matrix statistics for different taxonomic datasets. Matrix percentage equals the percent occupancy of species per locus. PI=parsimony informative sites.

\begin{tabular}{|c|c|c|c|c|c|c|c|c|}
\hline Dataset & $\begin{array}{l}\% \\
\text { Matrix }\end{array}$ & $\begin{array}{l}\# \\
\text { Loci }\end{array}$ & $\begin{array}{l}\text { \# Loci } \\
\text { (UCE / exon) }\end{array}$ & $\begin{array}{l}\text { Alignment } \\
\text { Length }\end{array}$ & $\begin{array}{l}\text { Mean Locus } \\
\text { Length ( } \pm \text { SD bp) }\end{array}$ & $\begin{array}{l}\text { Locus Length } \\
\text { Range (bp) }\end{array}$ & $\begin{array}{l}\text { \# PI } \\
\text { Sites }\end{array}$ & $\begin{array}{l}\% \text { PI } \\
\text { Sites }\end{array}$ \\
\hline Anthozoa+genome+outgroup* & 50 & 429 & $228 / 201$ & 81,403 & $190 \pm 89$ & $23-549$ & 40,041 & 49 \\
\hline \multirow{2}{*}{ Anthozoa } & 50 & 464 & $229 / 235$ & 91,455 & $197 \pm 93$ & $50-667$ & 43,501 & 48 \\
\hline & 25 & 1330 & $575 / 755$ & 254,596 & $191 \pm 99$ & $19-823$ & 109,930 & 43 \\
\hline Hexacorallia & 25 & 1052 & $529 / 523$ & 248,476 & $236 \pm 107$ & $52-1362$ & 63,968 & 26 \\
\hline \multirow{2}{*}{ Octocorallia } & 50 & 831 & $334 / 496$ & 208,869 & $251 \pm 127$ & $51-967$ & 70,369 & 34 \\
\hline & 25 & 1366 & $548 / 818$ & 368,275 & $270 \pm 132$ & $51-1013$ & 96,255 & 26 \\
\hline
\end{tabular}

* includes 33 taxa used in test run, 9 genome-enabled taxa, and the outgroup Hydra magnipapillata

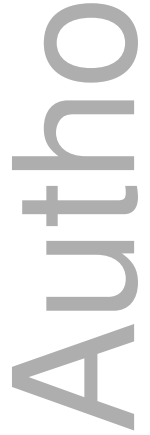

This article is protected by copyright. All rights reserved 
Table 4. Summary statistics for congeneric species alignments. Mean \% variation per locus is also included for UCE loci and exon

2 loci, respectively (in parentheses).

3

\begin{tabular}{|c|c|c|c|c|c|c|c|c|}
\hline & & & & Mean Locus & Locus & & Range $\%$ & Mean \% \\
\hline 1 & & \# Loci (UCE & Alignment & Length & Length & \# Variable & Variation per & Variation per \\
\hline Dataset & \# Loci & ( Exon) & Length & $( \pm$ SD bp $)$ & Range (bp) & Sites & Locus & Locus \\
\hline Acropora & 398 & $215 / 183$ & 206,067 & $517 \pm 73$ & $229-670$ & 9,474 & $0 *-46.0$ & $4.7(4.3,5.0)$ \\
\hline Alcyonium & 382 & $161 / 221$ & 205,676 & $538 \pm 250$ & $129-1470$ & 60,283 & $6.0-55.0$ & $30(28,31)$ \\
\hline Sinularia 3 & 426 & $162 / 264$ & 248,264 & $583 \pm 245$ & $91-1423$ & 14,231 & $0.3-27.0$ & $5.5(5.2,5.6)$ \\
\hline
\end{tabular}

* Only one locus was not polymorphic



This article is protected by copyright. All rights reserved 


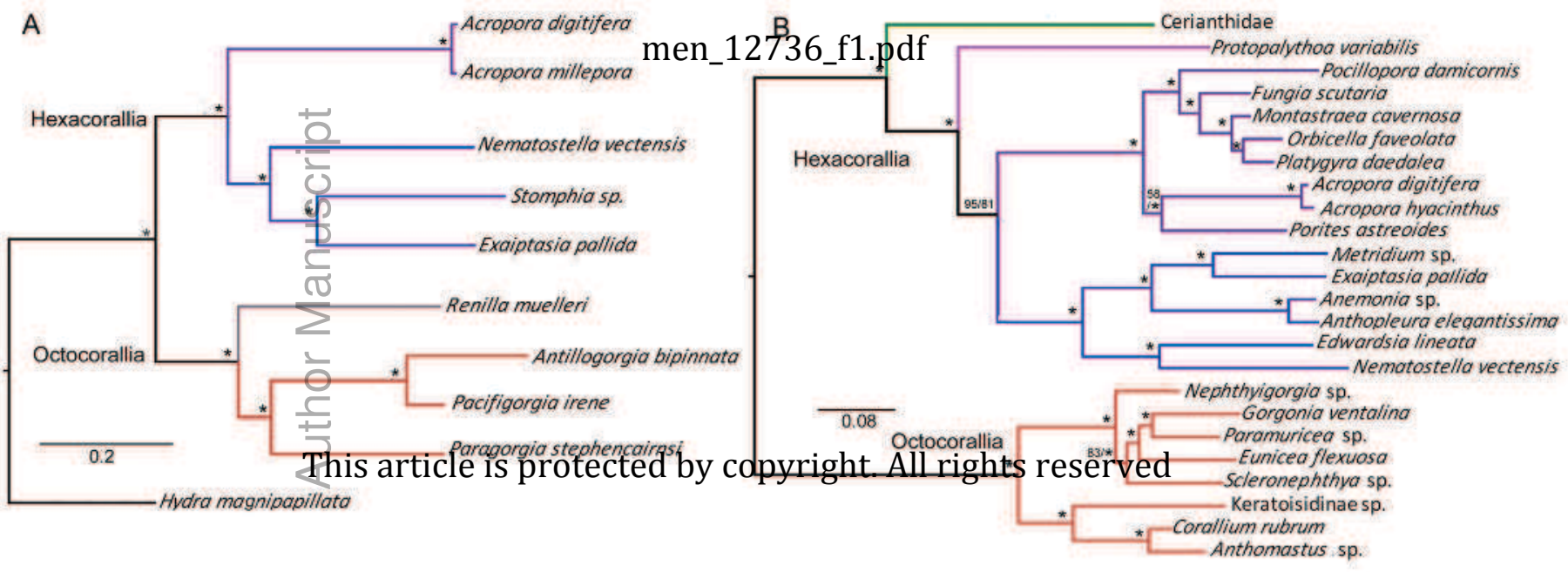




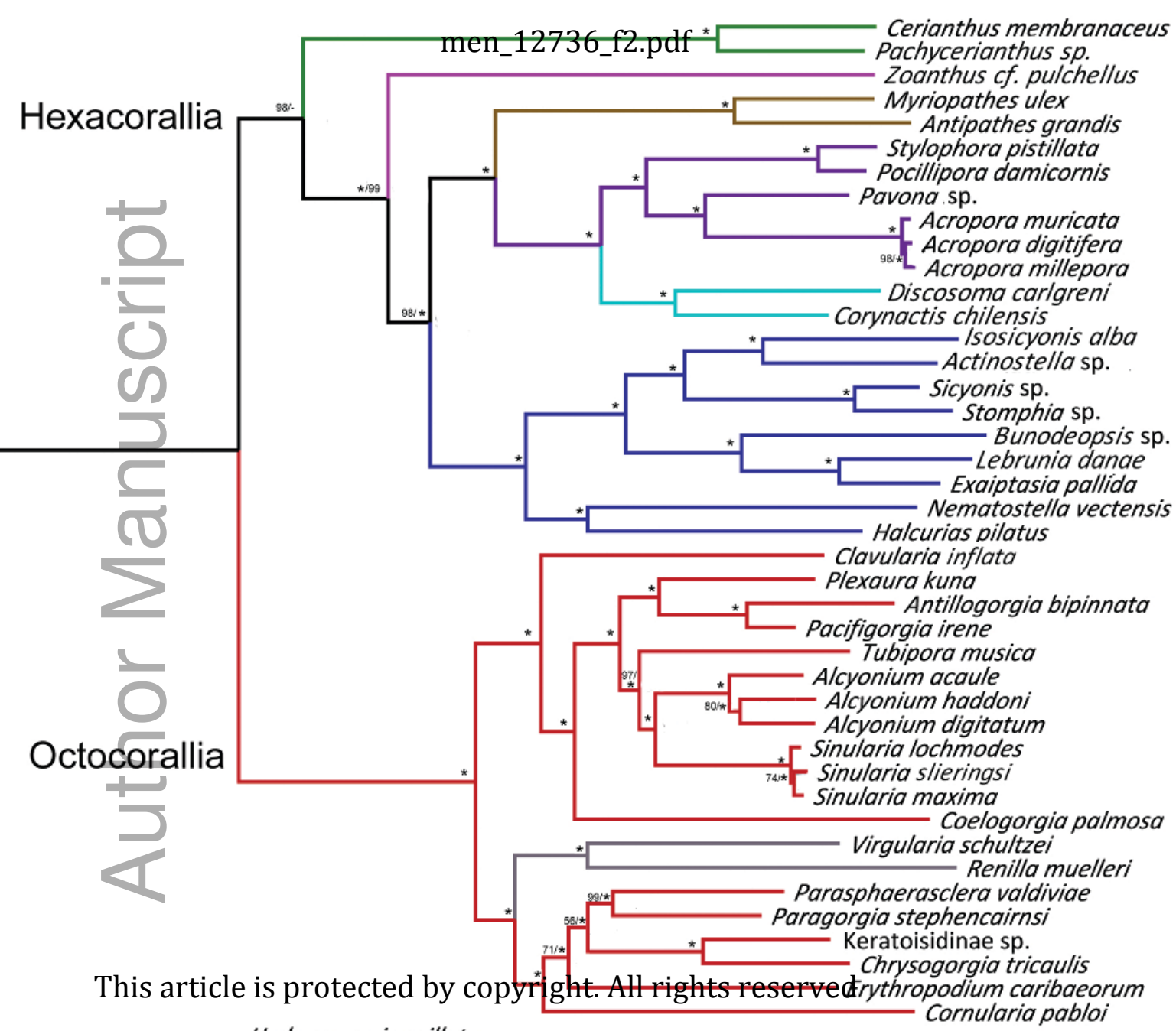

Hydra magnipapillata 